\title{
Population-based outcome of muscle-invasive bladder cancer following radical cystectomy: who can benefit from adjuvant chemotherapy?
}

\author{
Junjie Tian\#, Junjie Sun", Guanghou Fu, Zhijie Xu, Xiaoyi Chen, Yue Shi, Baiye Jin^ \\ Department of Urology, The First Affiliated Hospital, School of Medicine, Zhejiang University, Hangzhou, China \\ Contributions: (I) Conception and design: J Tian, J Sun; (II) Administrative support: None; (III) Provision of study materials or patients: None; (IV) \\ Collection and assembly of data: Y Shi, X Chen; (V) Data analysis and interpretation: G Fu, Z Xu; (VI) Manuscript writing: All authors; (VII) Final \\ approval of manuscript: All authors. \\ \#These authors contributed equally to this work. \\ Correspondence to: Dr. Baiye Jin. Department of Urology, The First Affiliated Hospital, School of Medicine, Zhejiang University, 79 Qingchun Road, \\ Hangzhou 310003, China. Email: jinbaiye1964@zju.edu.cn.
}

Background: The benefit of adjuvant chemotherapy remains controversial in muscle-invasive bladder cancer (MIBC) after radical cystectomy. The present study's primary objective was to construct a predictive tool for the reasonable application of adjuvant chemotherapy.

Methods: All of the patients analyzed in the present study were recruited from the Surveillance Epidemiology and End Results program between 2004 and 2015. Propensity score matching (PSM) was used to reduce inherent selection bias. Cox proportional hazards models were applied to identify the independent prognostic factors of overall survival (OS) and cancer-specific survival (CSS), which were further used to construct prognostic nomogram and risk stratification systems to predict survival outcomes. The prognostic nomogram's performance was assessed by concordance index (C-index), receiver-operating characteristic (ROC) and calibration curves. Decision curve analysis (DCA) was performed to evaluate the clinical net benefit of the prognostic nomogram.

Results: A total of 6,384 patients with or without adjuvant chemotherapy were included after PSM. Several independent predictors for OS and CSS were identified and further applied to establish a nomogram for 3-, 5- and 10-year, respectively. The nomogram showed favorable discriminative ability for the prediction of OS and CSS, with a C-index of 0.709 [95\% confidence interval (CI): 0.699-0.719] for OS and 0.728 (95\% CI: 0.718-0.738) for CSS. ROC and calibration curves showed satisfactory consistency. The DCA revealed high clinical positive net benefits of the prognostic nomogram. The different risk stratification systems showed that adjuvant chemotherapy resulted in better OS $(\mathrm{P}<0.001)$ and CSS $(\mathrm{P}<0.001)$ than without adjuvant chemotherapy for high-risk patients; while the OS $(\mathrm{P}=0.350)$ and CSS $(\mathrm{P}=0.260)$ for low-risk patients were comparable.

Conclusions: We have constructed a predictive model and different risk stratifications for selecting a population that could benefit from postoperative adjuvant chemotherapy. Adjuvant chemotherapy was found to be beneficial for high-risk patients, while low-risk patients should be carefully monitored.

Keywords: Muscle-invasive bladder cancer (MIBC); radical cystectomy; adjuvant chemotherapy; predictive tool

Submitted Jun 01, 2020. Accepted for publication Nov 03, 2020.

doi: $10.21037 /$ tau-20-960

View this article at: http://dx.doi.org/10.21037/tau-20-960

^ ORCID: 0000-0002-6667-0424. 


\section{Introduction}

Bladder cancer (BC) is the ninth most commonly diagnosed malignancy and ranks 13th in terms of the causes of death worldwide (1). It has been reported that approximately $20-30 \%$ of patients have muscle-invasive bladder cancer (MIBC), which is a poor prognostic stage of BC that requires radical surgical treatment or chemo-radiation with intensive surveillance (2-4). Radical cystectomy (RC) with extended pelvic lymph node dissection is the gold standard care for MIBC, and is an effective therapy to improve the survival rate avoid locoregional recurrence and distant metastasis for these patients (5-7). Despite RC, approximately $50 \%$ of MIBC patients will develop metastasis in the first 2-3 years of diagnosis and finally succumb due to pre-existing metastatic disease or locoregional recurrence $(8,9)$. These survival outcomes indicated that $\mathrm{RC}$ alone may not be completely effective for controlling the disease, and that the extended use of systemic therapies should be considered in most locally advanced MIBC patients (10).

With the development of multidisciplinary approaches for BC, the application of perioperative (neoadjuvant or adjuvant) systemic chemotherapy combined with $\mathrm{RC}$ has been intensively studied. Therefore, according to current international guidelines, the use of neoadjuvant cisplatinbased chemotherapy is recommended as level of evidence one $(11,12)$. In clinical practice, neoadjuvant chemotherapy has been used sparingly for various reasons, and adjuvant treatment is used more frequently (13-16). Although there have been several observational retrospective studies $(17,18)$, meta-analyses $(10,19)$, and randomized clinical trials $(20,21)$ evaluating the survival benefits of adjuvant chemotherapy on MIBC patients following $\mathrm{RC}$, the routine use of adjuvant chemotherapy for MIBC management remains controversial. Some patients may benefit from adjuvant chemotherapy, however, it is unclear which patients will benefit or how to select suitable patients for adjuvant chemotherapy.

It is generally perceived that not all MIBC patients after RC should receive adjuvant chemotherapy, but that this should be limited to patients at high risk of recurrence. MIBC patients receive with or without adjuvant chemotherapy following RC include those with pT3-4 stages, lymph node involvement, a lymph node density $>20 \%$, and positive surgical margins, which are commonly recognized as poor prognostic factors $(11,22,23)$. Although some previously published studies have assessed the risk factors for recurrence and death in MIBC patients, and established prognostic models according to important risk factors, these models mainly revealed that the low survival rate of high-risk patients and the need for more attention, but they did not provide specific and intuitive personalized therapy for each patient (15,24-27).

The primary objective of the present study was to use the Surveillance Epidemiology and End Results (SEER) program to construct a predictive model and different risk stratification systems, determine the risk cutoff point, select a population that could benefit from postoperative adjuvant chemotherapy. We hope this predictive tool could provide an individualized and accurate reference for the reasonable application of adjuvant chemotherapy. We present the following article in accordance with the TRIPOD reporting checklist (available at http://dx.doi.org/10.21037/ tau-20-960).

\section{Methods}

\section{Data source}

The study participants were recruited from the SEER database of the National Cancer Institute (NCI) from 2004 to 2015, which is publicly accessible and covers approximately $30 \%$ of the US population (https://www. seer.cancer.gov/) (28). We used the SEER*Stat version 8.3.6 software to determine the eligible population from the Incidence-SEER18 Regs Custom Data, Nov2018 Sub (1975-2016 varying). The inclusion criteria were as follows: (I) microscopically diagnosed with primary BC as the first malignancy from 2004 to 2015; (II) confirmed to have MIBC (pT2-T4); (III) histological subtypes were coded by the International Classification of Diseases for Oncology, 3rd Edition (ICD-O-3) limited to bladder transitional cell carcinoma (ICD-O-3: 8120/3, 8122/3, 8130/3, 8131/3); and (IV) BC patients underwent RC (codes 40, 50, 60, and 70 in the site-specific surgery variable, and the codes 50 , $60-64,70-74$, and 80 in the summary variable) (29-31). A total of 10847 cases were initially eligible for inclusion. The following exclusion criteria were identified: (I) missing or incomplete clinical information, such as race, American Joint Committee on Cancer (AJCC) TNM staging system, regional nodes examined status and number, regional nodepositive status and number, survival times, and cause of death; (II) patients who had received pre-operative, intraoperative or post-operative radiotherapy. (III) age $<18$ years, (IV) distant metastases (M1 stage), and (V) survival time 


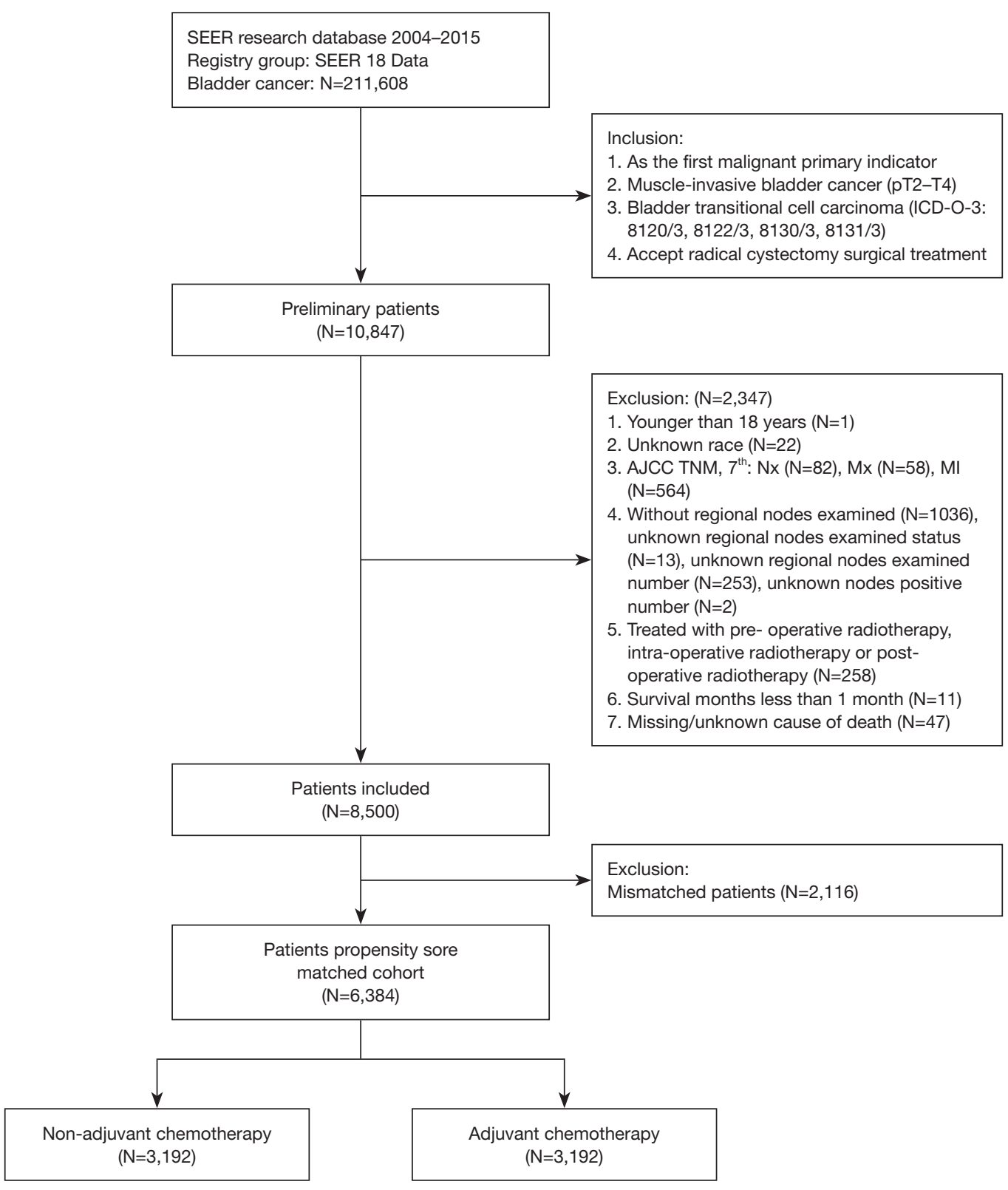

Figure 1 The flow chart of muscle-invasive bladder cancer patients' selection. AJCC, American Joint Committee on Cancer; ICD-O-3, International Classification of Disease for Oncology, Third Edition; SEER, Surveillance Epidemiology and End Results; TNM, tumornode-metastasis.

$<1$ month. The detailed eligible population selection process is shown in Figure 1. Propensity score matching (PSM) of the adjuvant chemotherapy and non-adjuvant chemotherapy groups was used to reduce inherent selection bias, 2,116 mismatched patients were excluded, and a total of 6,384 patients were finally included for further analyses. The study has conformed to the provisions of the Declaration of Helsinki (as revised in 2013). The data analyzed in our current study accessed from SEER database (https://www. seer.cancer.gov/), which is publicly available and all patient data are de-identified. We obtained the permission to access the SEER program with the ID number 17051-Nov2018 via Internet access method. Ethical approval was waived by the local ethics committee. 


\section{Endpoints}

The primary endpoint was overall survival (OS), while cancer-specific survival (CSS) was a secondary endpoint. Survival time (in months) was set as the time from BC diagnosis to death or date of last follow-up time (the specific definition process of disease survival time in the SEER database can be obtained at: https://seer.cancer.gov/ survivaltime/3-fields-survival-time-active.pdf). The final study population were included 8500 cases diagnosed with MIBC who underwent RC between 2004 and 2015, these patients were followed-up to December 31, 2015.

\section{Variables}

Patients' demographic and clinical-pathological characteristics were considered for further analyses, including age, gender, race, pathological grade, $\mathrm{pT}$ stage, pN stage, lymph nodes harvested (LNH) count, lymph nodes ratio (LNR), tumor size, adjuvant chemotherapy, follow-up time, and cause of death. Patients were grouped as $<65,65-75$, and $>75$ years according to age at diagnosis; race was classified as Black, White and Others (American Indian/AK Native, Asian/Pacific Islander); pathological grade was categorized into well/moderately differentiated (I/II), poorly differentiated (III), undifferentiated (IV), and unknown, based on SEER codes (32); and TNM stage was regrouped based on the AJCC $\left(7^{\text {th }}\right.$ edition) staging system codes, respectively. It was reported that $10 \mathrm{LNH}$ was related to survival outcomes (33-35), in line with prior reports $(31,32), \mathrm{LNH}$ was categorized into $<10$, and $\geq 10$, respectively. LNR was calculated as the number of positive nodes to the number of nodes harvested, which was grouped using previously described cutoffs of $0 \%, 0.1-20 \%$, and $20 \%$ (36-38). Tumor size was divided into $<50 \mathrm{~mm}$, $\geq 50 \mathrm{~mm}$, and unknown (30); adjuvant chemotherapy was denoted as none and yes according to SEER codes.

\section{Statistical analysis}

PSM is a statistical tool that matches patients with one or more control cases according to their propensity scores, reducing inherent selection bias (39). The matching ratio was $1: 1$, and the caliper applied for matching was set at 0.1 . The $\chi^{2}$-test was used to contrast the baseline characteristics between cohorts before and after PSM for the adjuvant chemotherapy and non-adjuvant chemotherapy groups.
Univariate and multivariate Cox regression models were performed to determine the independent prognostic variables of OS and CSS, which were further applied to construct a prognostic nomogram and risk stratification systems to predict survival probabilities. The nomogram's validation was performed by using the C-index, ROC curves (40), calibration curves, and decision curve analysis (DCA) (41). Concordance index (C-index) and receiver-operating characteristic (ROC) curves analyses were used to assess the predictive accuracy and discrimination ability of prognostic nomogram. Calibration curves (1,000 bootstrap resamples) were employed to measure the calibration of prognostic nomogram (42). DCA was used to assess clinical positive net benefits of the prognostic nomogram. Furthermore, to verify independent discriminatory ability of the prognostic nomogram, the low- and high-risk stratification systems for OS and CSS were constructed according to the total risk scores of the established nomogram (grouped by the cutoff values of the median score). The log-rank test and KaplanMeier curves were further applied to determine the OS and CSS of eligible MIBC patients with or without adjuvant chemotherapy in different prognostic risk stratification systems. R version 3.6.1 software (Institute for Statistics and Mathematics, Vienna, Austria; https://www.r-project.org/) was used to perform all statistical analyses. $\mathrm{P}<0.05$ indicated statistical significance.

\section{Results}

\section{Patients' baseline characteristics}

The present study included 8500 eligible patients diagnosed with MIBC following RC without distant metastasis from 2004 to 2015 from the SEER program. A total of 3,809 patients received adjuvant chemotherapy, and 4,691 patients did not. A total of 2,098 mismatched patients were excluded after the PSM was utilized. Finally, 6,384 patients were divided into non-adjuvant chemotherapy $(\mathrm{n}=3,192)$ and adjuvant chemotherapy $(n=3,192)$ for further analyses. The baseline demographics and clinical-pathological characteristics of eligible patients before and after PSM are summarized in Table 1. The histograms of propensity scores before and after matching are shown in Figure S1. The median follow-up time was 32 months (range: $1-$ 155 months). A total of 4,441 (52.2\%) patients had died by the last follow-up date, of which 3,273 (73.7\%) had died from MIBC and 1,168 (26.3\%) from other causes. 
Table 1 Baseline clinicopathological characteristics and treatment experience of all patients with muscle-invasive bladder cancer

\begin{tabular}{|c|c|c|c|c|c|c|}
\hline Characteristics & \multicolumn{3}{|c|}{ Before PSM } & \multicolumn{3}{|c|}{ After PSM } \\
\hline Age (years) & & & $<0.001$ & & & 0.002 \\
\hline$<65$ & $1,586(33.8)$ & $1,892(49.7)$ & & $1,406(44.0)$ & 1,395 (43.7) & \\
\hline$>75$ & $1,379(29.4)$ & $492(12.9)$ & & $586(18.4)$ & $492(15.4)$ & \\
\hline Gender & & & 0.694 & & & 0.126 \\
\hline Female & $1,118(23.8)$ & $893(23.4)$ & & 730 (22.9) & $783(24.5)$ & \\
\hline Black & $293(6.2)$ & $216(5.7)$ & & $211(6.6)$ & $180(5.6)$ & \\
\hline Others & $245(5.2)$ & $197(5.2)$ & & $140(4.4)$ & $182(5.7)$ & \\
\hline Pathological grade & & & $<0.001$ & & & 0.091 \\
\hline $\mathrm{I} / \mathrm{II}$ & 155 (3.3) & $69(1.8)$ & & $75(2.3)$ & $68(2.1)$ & \\
\hline III & $1,517(32.3)$ & $938(24.6)$ & & $864(27.1)$ & 892 (27.9) & \\
\hline IV & $2,835(60.4)$ & 2,650 (69.6) & & $2,092(65.5)$ & 2,127 (66.6) & \\
\hline Unknown & $184(3.9)$ & $152(4.0)$ & & $161(5.0)$ & 105 (3.3) & \\
\hline No & 3,701 (78.9) & 2,391 (62.8) & & $2,344(73.4)$ & $2,216(69.4)$ & \\
\hline N1 & $527(11.2)$ & 703 (18.5) & & 417 (13.1) & $553(17.3)$ & \\
\hline N2 & $445(9.5)$ & $696(18.3)$ & & 413 (12.9) & 411 (12.9) & \\
\hline N3 & $18(0.4)$ & $19(0.5)$ & & $18(0.6)$ & $12(0.4)$ & \\
\hline LNH & & & $<0.001$ & & & 0.396 \\
\hline$<10$ & $1,749(37.3)$ & 998 (26.2) & & $936(29.3)$ & $968(30.3)$ & \\
\hline$\geq 10$ & $2,942(62.7)$ & $2,811(73.8)$ & & $2,256(70.7)$ & $2,224(69.7)$ & \\
\hline LNR & & & $<0.001$ & & & 0.017 \\
\hline $0 \%$ & $3,704(79.0)$ & $2,450(64.3)$ & & $2,346(73.5)$ & 2,264 (70.9) & \\
\hline $0.1-20 \%$ & $517(11.0)$ & 819 (21.5) & & $453(14.2)$ & $534(16.7)$ & \\
\hline$>20 \%$ & $470(10.0)$ & $540(14.2)$ & & 393 (12.3) & 394 (12.3) & \\
\hline
\end{tabular}

Table 1 (continued) 
Table 1 (continued)

\begin{tabular}{|c|c|c|c|c|c|c|}
\hline Characteristics & \multicolumn{3}{|c|}{ Before PSM } & \multicolumn{3}{|c|}{ After PSM } \\
\hline Tumor size (mm) & & & 0.152 & & & 0.172 \\
\hline$<50$ & $2,271(48.4)$ & $1,792(47.0)$ & & $1,531(48.0)$ & $1,440(45.1)$ & \\
\hline Unknown & $1,071(22.8)$ & 848 (22.3) & & $738(23.1)$ & $785(24.6)$ & \\
\hline
\end{tabular}

LNH, lymph nodes harvested; LNR, lymph nodes ratio; PSM, propensity score matching.

\section{Screening of significant prognostic factors for OS and CSS using univariable and multivariable analyses}

The Cox proportional hazards models were used to determine the independent prognostic factors in the prediction of OS and CSS. Univariable analyses showed that a total 10 factors were related to OS and CSS. These were as follows: age, gender, race, pathological grade, $\mathrm{pT}$ stage, $\mathrm{pN}$ stage, LNH, LNR, tumor size, and adjuvant chemotherapy (Tables 2 and 3). Multivariable Cox analyses indicated that age, race, $\mathrm{pT}$ stage, $\mathrm{pN}$ stage, $\mathrm{LNH}, \mathrm{LNR}$, tumor size, and adjuvant chemotherapy were significant independent prognostic factors of OS (Table 2). Except pathological grade, these comparable variables were also significant prognostic risk factors for CSS (Table 3). Significant independent prognostic factors were integrated into the predictive models for nomogram and risk stratification systems.

\section{Establishment and validation of the novel prognostic nomogram}

A prognostic nomogram for predicting 3-, 5-, and 10-year OS and CSS in MIBC patients in the entire cohorts after PSM was developed (Figure 2). These predictive tools were established to predict the probabilities of 3-, 5-, and 10-year OS and CSS for MIBC patients by calculating the total points of each significant prognostic factors. The performance of the prognostic nomogram was further validated for discrimination and calibration. The prognostic nomogram C-index for the prediction of OS and CSS was 0.709 (95\% CI: 0.699-0.719) and 0.728 (95\% CI: 0.718 0.738), respectively, demonstrating relatively good model discriminative ability. Besides, time-dependent ROC analyses for 3-, 5-, and 10-year OS and CSS were used to validate the prognostic nomogram, which revealed good discrimination in predicting the prognosis of MIBC patients (Figure S2A,B). Calibration curves for OS and CSS probabilities of 3-, 5-, and 10-year showed satisfactory consistency of the constructed prognostic nomogram (Figure S2C,D). Moreover, DCA was used to assess the effectiveness and advantages of predictive models, which identify and compare the clinical application value for the prognostic nomogram by calculating the clinical positive net benefits among all of the threshold probabilities at 3-, 5-, and 10-year time points. Accordingly, the high clinical positive net benefits were achieved by assessing OS and CSS at different threshold probabilities in our prognostic nomogram (Figure 3).

\section{Comparisons of OS and CSS for patients in different risk stratification systems}

To determine the independent discrimination of the novel prognostic nomogram, the low- and high-risk stratification systems for OS and CSS were constructed according to the total risk scores of the established nomogram (grouped by the cutoff values of the median score). The cohort was divided into the low-risk $(3,107 / 6,384,48.7 \%$, score $0-120.1)$, and high-risk groups $(3,227 / 6,384,51.3 \%$, score $\geq 120.1)$ for OS, and into the low-risk $(3,191 / 6,384,50.0 \%$, score $0-106.3)$, and high-risk groups $(3,193 / 6,384,50.0 \%$, score $\geq 106.3$ ) for CSS. Furthermore, the log-rank test and Kaplan-Meier curves were further applied to determine the OS and CSS of MIBC patients in different prognostic risk stratification systems, the prognostic nomogram could discriminate MIBC patients with different risk stratification systems (Figure 4). Group adjuvant chemotherapy showed significantly better OS $(\mathrm{P}<0.001)$ and CSS $(\mathrm{P}=0.033)$ than group non-adjuvant chemotherapy before risk stratification (Figure $5 A, B$ ). Furthermore, the different risk stratification 
Table 2 Univariate and multivariate analyses of prognostic factors associated with overall survival of patients with MIBC

\begin{tabular}{|c|c|c|c|c|}
\hline Characteristics & \multicolumn{2}{|c|}{ Univariate analysis } & \multicolumn{2}{|c|}{ Multivariate analysis } \\
\hline$<65$ & 1 (ref) & & 1 (ref) & \\
\hline $65-75$ & $1.261(1.166-1.364)$ & $<0.001$ & $1.267(1.171-1.371)$ & $<0.001$ \\
\hline \multicolumn{5}{|l|}{ Gender } \\
\hline Female & 1 (ref) & & NA & NA \\
\hline Male & $0.946(0.872-1.026)$ & 0.180 & NA & NA \\
\hline Others & $0.922(0.782-1.087)$ & 0.331 & $0.885(0.750-1.044)$ & 0.148 \\
\hline \multicolumn{5}{|c|}{ Pathological grade } \\
\hline $1 / I I$ & 1 (ref) & & & \\
\hline III & $1.246(0.979-1.585)$ & 0.074 & NA & NA \\
\hline IV & $1.116(0.880-1.413)$ & 0.365 & NA & NA \\
\hline Unknown & $1.021(0.760-1.371)$ & 0.893 & NA & NA \\
\hline \multicolumn{5}{|l|}{ pT stage } \\
\hline NO & 1 (ref) & & 1 (ref) & \\
\hline $\mathrm{N} 1$ & $2.310(2.115-2.523)$ & $<0.001$ & $0.901(0.556-1.460)$ & 0.673 \\
\hline N2 & $3.548(3.242-3.883)$ & $<0.001$ & 1.175 (0.724-1.907) & 0.513 \\
\hline N3 & $6.456(4.440-9.390)$ & $<0.001$ & 2.002 (1.087-3.686) & 0.026 \\
\hline \multicolumn{5}{|l|}{ LNH } \\
\hline$<10$ & 1 (ref) & & 1 (ref) & \\
\hline$\geq 10$ & $0.772(0.718-0.830)$ & $<0.001$ & $0.832(0.771-0.898)$ & $<0.001$ \\
\hline \multicolumn{5}{|l|}{ LNR } \\
\hline $0 \%$ & 1 (ref) & & 1 (ref) & \\
\hline $0.1-20 \%$ & $2.360(2.161-2.577)$ & $<0.001$ & $1.970(1.215-3.194)$ & 0.006 \\
\hline$>20 \%$ & $3.779(3.455-4.133)$ & $<0.001$ & 2.539 (1.564-4.122) & $<0.001$ \\
\hline
\end{tabular}

Table 2 (continued) 
Table 2 (continued)

\begin{tabular}{|c|c|c|c|c|}
\hline Characteristics & \multicolumn{2}{|c|}{ Univariate analysis } & \multicolumn{2}{|c|}{ Multivariate analysis } \\
\hline$<50$ & 1 (ref) & & 1 (ref) & \\
\hline$\geq 50$ & $1.402(1.296-1.518)$ & $<0.001$ & $1.280(1.181-1.387)$ & $<0.001$ \\
\hline \multicolumn{5}{|c|}{ Adjuvant chemotherapy } \\
\hline No & 1 (ref) & & 1 (ref) & \\
\hline Yes & $0.851(0.794-0.913)$ & $<0.001$ & $0.731(0.681-0.784)$ & $<0.001$ \\
\hline
\end{tabular}

LNH, lymph nodes harvested; LNR, lymph nodes ratio; MIBC, muscle-invasive bladder cancer.

Table 3 Univariate and multivariate analyses of prognostic factors associated with cancer-specific survival of patients with MIBC

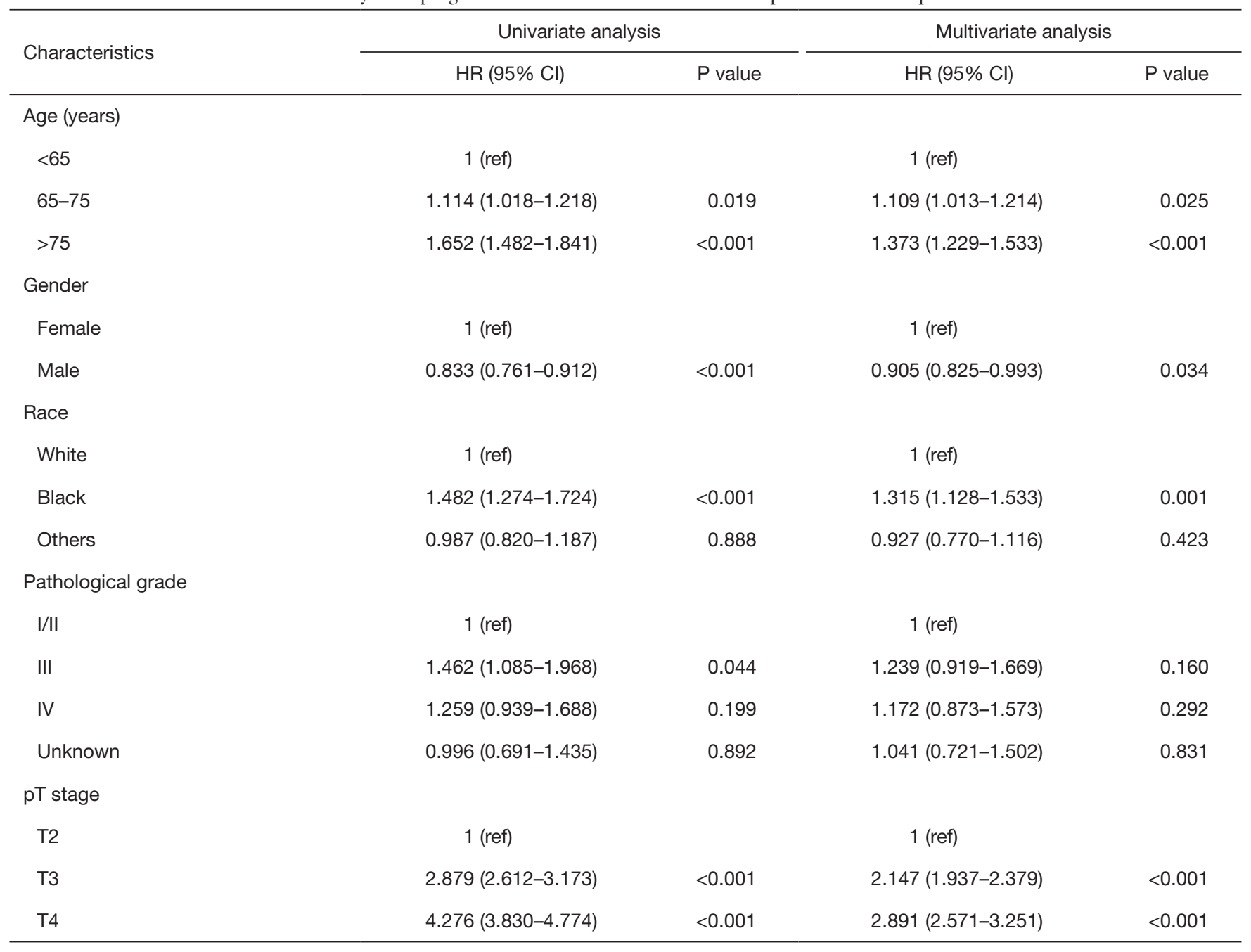

Table 3 (continued) 
Table 3 (continued)

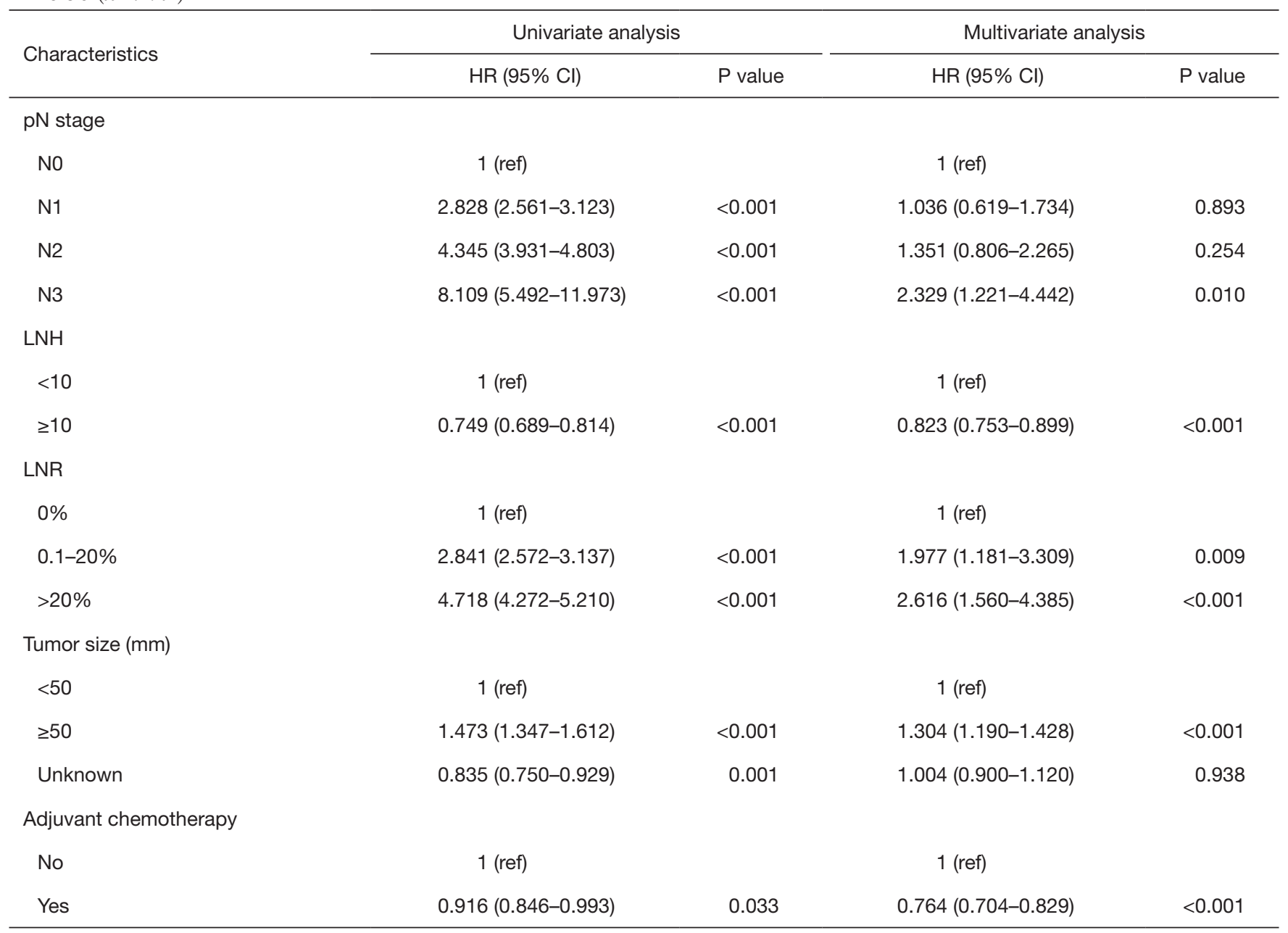

LNH, lymph nodes harvested; LNR, lymph nodes ratio; MIBC, muscle-invasive bladder cancer.

subgroups showed that adjuvant chemotherapy had better OS $(\mathrm{P}<0.001)$ and CSS $(\mathrm{P}<0.001)$ than without adjuvant chemotherapy for high-risk patients (Figure 5C,D); while the OS $(\mathrm{P}=0.350)$ and $\operatorname{CSS}(\mathrm{P}=0.260)$ for low-risk patients were comparable (Figure $5 E, F)$.

\section{Discussion}

RC with urinary diversion and pelvic lymph nodes dissection is the gold standard treatment for MIBC patients $(5,43)$. It includes the removal of micrometastases to prevent local recurrence and increase the chance of healing, thereby achieving local tumor control $(44,45)$. Despite these advantages, the rate of postoperative complications in $\mathrm{RC}$ is $31-51 \%$, and approximately half of MIBC patients will develop metastasis in the first 2-3 years after diagnosis and finally succumb due to pre-existing metastatic disease or locoregional recurrence $(8,9,46,47)$. This highlights the need for effective adjuvant therapy in the treatment of MIBC to eradicate subclinical diseases that may be arise at the time of diagnosis, thereby improving survival outcomes. There have been significant advances in adjuvant therapy in recent years, and several researches have assessed and validated the curative effect of neoadjuvant or adjuvant systemic chemotherapies before or after surgical resection $(12,20,21,48,49)$. However, which patients will benefit or how to select the most suitable patients for adjuvant chemotherapy remains controversial. Therefore, we carried out an in-depth quantitative exploration of the prognostic factors of MIBC patients using a nomogram. We constructed a predictive model and different risk stratification systems, determined the risk cutoff point, 
A

Points

Age (years)

Race

pT.stage

pN.stage

LNH

LNR

Tumor.size (mm)

Total points

Linear Predictor

3-year survival probability of OS

5-year survival probability of OS

10-year survival probability of OS

B

Points

Age (years)

Gender

Race

pT.stage

pN.stage

LNH

LNR

Tumor.size (mm)

Total points

Linear Predictor

3-year survival probability of CSS

5-year survival probability of CSS

10-year survival probability of CSS
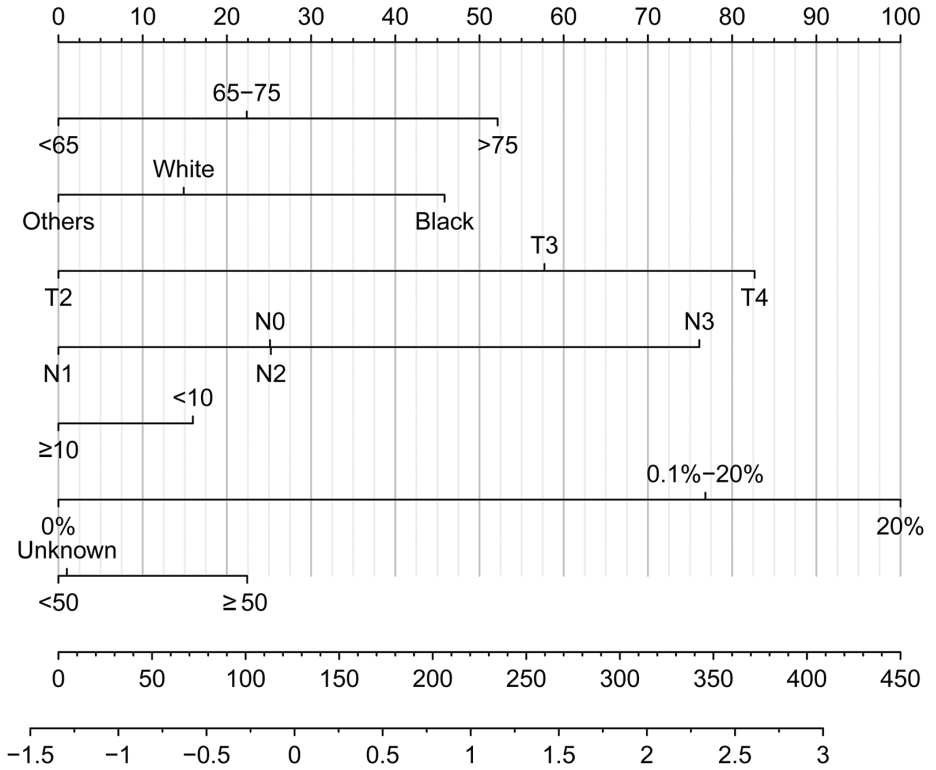

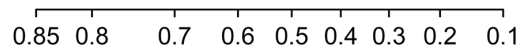

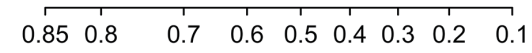

\begin{tabular}{llllllll}
\hline 0.8 & 0.7 & 0.6 & 0.5 & 0.4 & 0.3 & 0.2 & 0.1
\end{tabular}
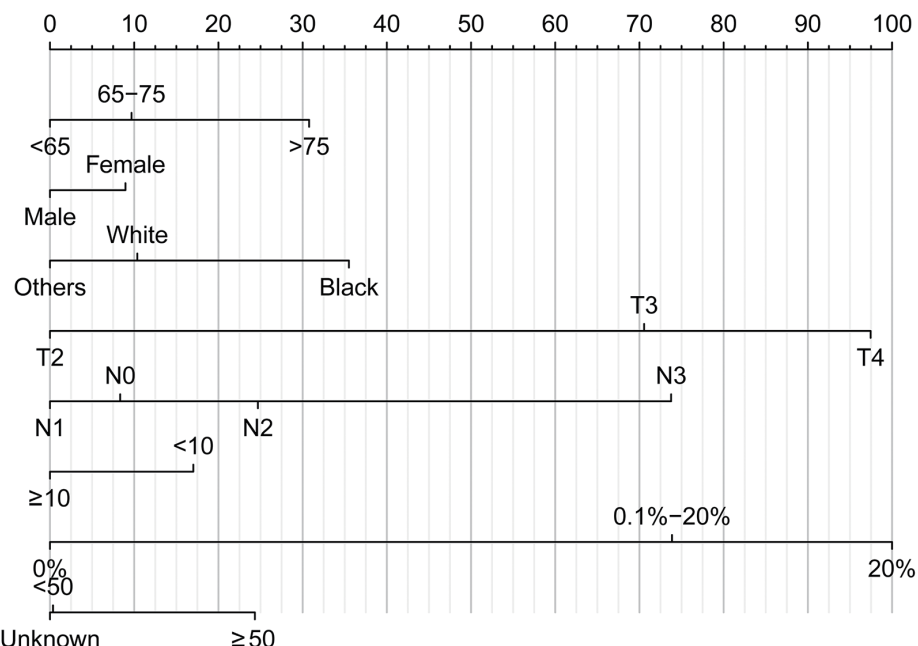

Unknown $\quad \geq 50$

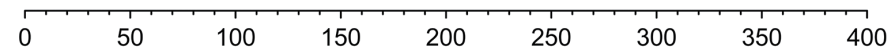

\begin{tabular}{llllllllll}
\hline-1.5 & -1 & -0.5 & 0 & 0.5 & 1 & 1.5 & 2 & 2.5 & 3
\end{tabular}

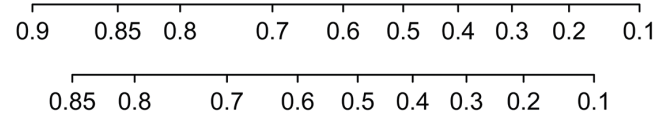

$\begin{array}{lllllllll}0.85 & 0.8 & 0.7 & 0.6 & 0.5 & 0.4 & 0.3 & 0.2 & 0.1\end{array}$

Figure 2 The nomogram for predicting 3-, 5-, and 10-year probabilities of OS (A) and CSS (B) in patients with MIBC. LNH, lymph nodes harvested; LNR, lymph nodes ratio; MIBC, muscle-invasive bladder cancer; OS, overall survival; CSS, cancer-specific survival. 

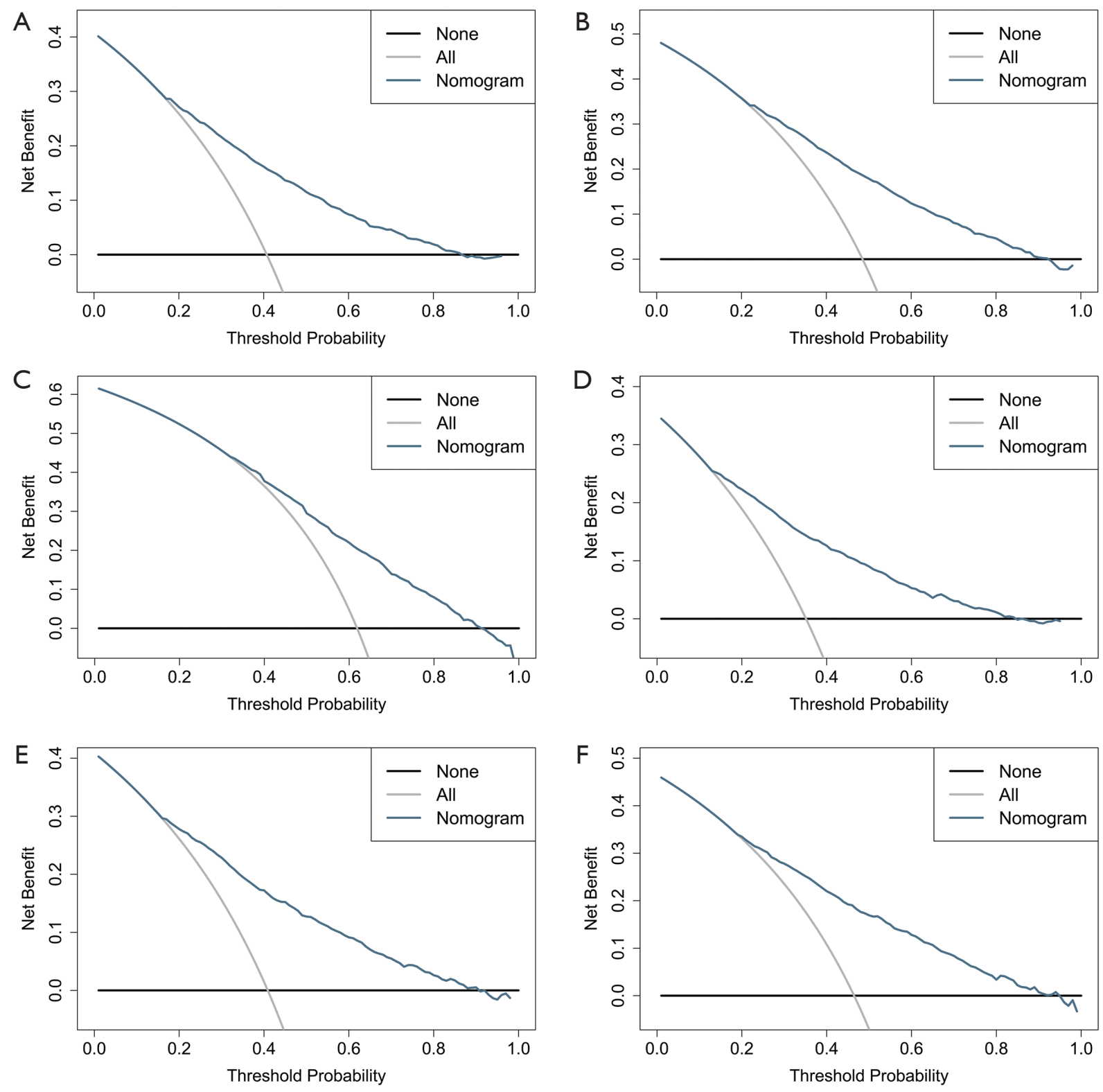

Figure 3 Decision curves of the nomogram predicting OS and CSS. (A,B,C) The 3-, 5-, and 10-year OS nomograms decision curves, respectively; (D,E,F) the 3-, 5-, and 10-year CSS nomograms decision curves, respectively. The X-axis represents the threshold probabilities, and the Y-axis measures the net benefit calculated by adding the true positives and subtracting the false positives. The horizontal black line along the $\mathrm{X}$-axis assumes that death occurred in no patients, whereas the solid gray line assumes that all patients will have death at a specific threshold probability. The blue color solid line represents the clinical positive net benefit of using the prognostic nomogram. OS, overall survival; CSS, cancer-specific survival.

selected a population that could benefit from postoperative adjuvant chemotherapy, and provided a guide for further follow-up strategies.

In our current large retrospective cohorts, recruited from the SEER program, several demographic and clinical- pathological factors were found to be significantly related to the prognosis of OS and CSS, including age, gender, race, $\mathrm{pT}$ stage, $\mathrm{pN}$ stage, $\mathrm{LNH}, \mathrm{LNR}$, and tumor size. In the present study, approximately $60 \%$ of MIBC patients were $>65$ years, these patients had poorer OS and CSS than 

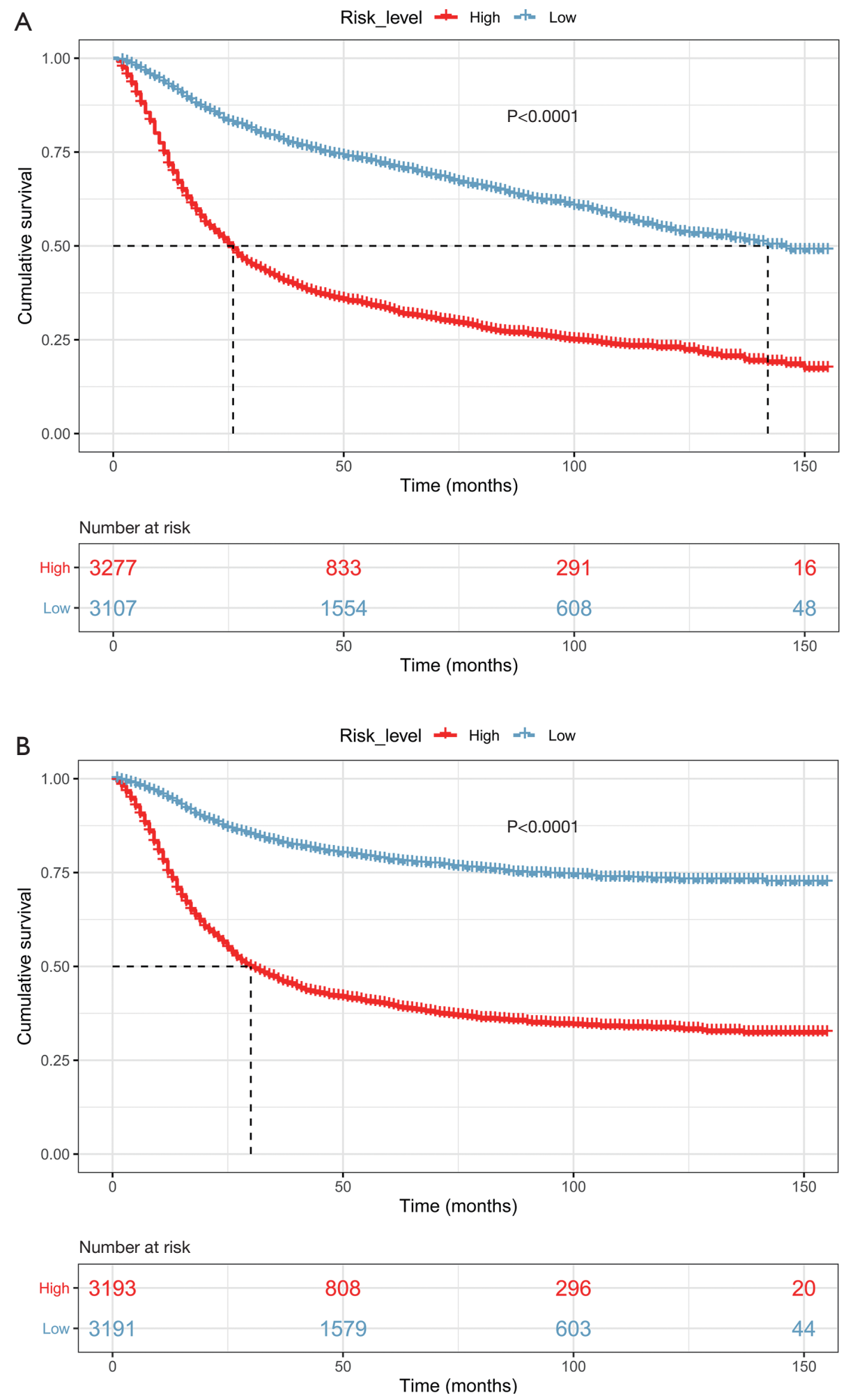

Figure 4 Kaplan-Meier curves of OS and CSS in the different risk stratification systems. (A) The comparisons of OS for low- and high-risk groups patients; (B) the comparisons of CSS for low- and high-risk groups patients. OS, overall survival; CSS, cancer-specific survival. 
A
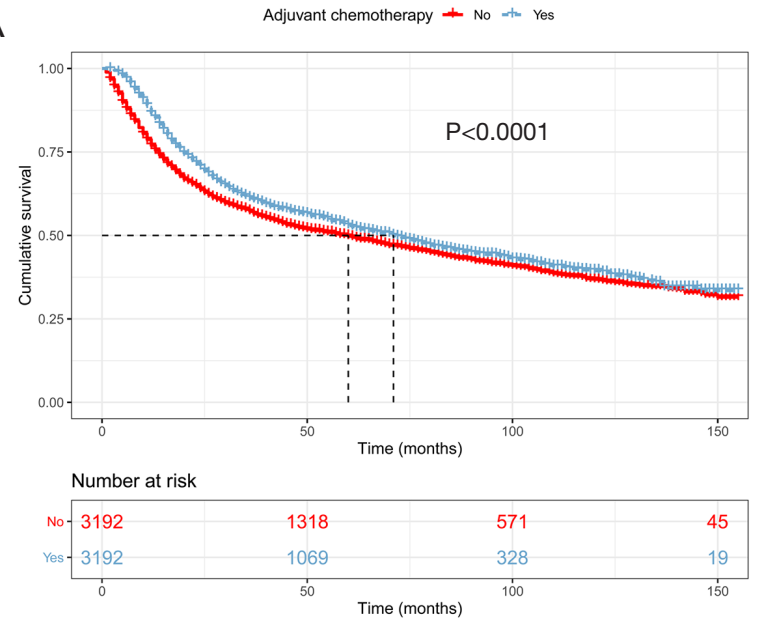

C

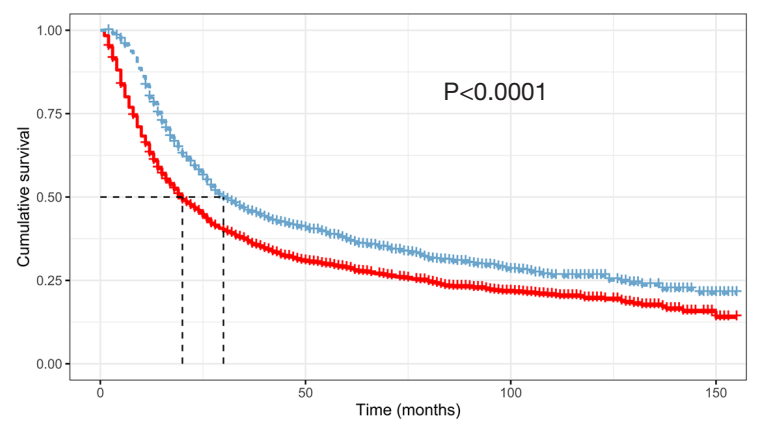

Number at risk

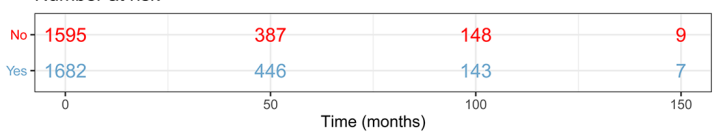

$\mathrm{E}$
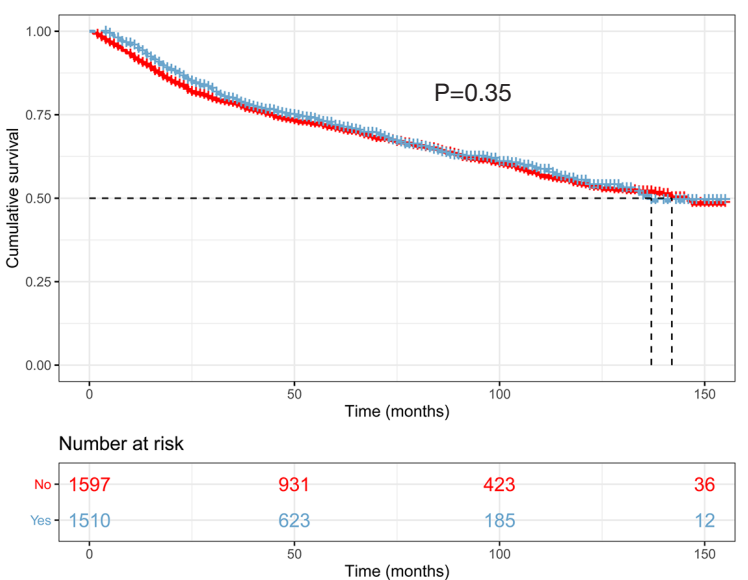

B Adjuvant chemotherapy + No $=$ tw Yes
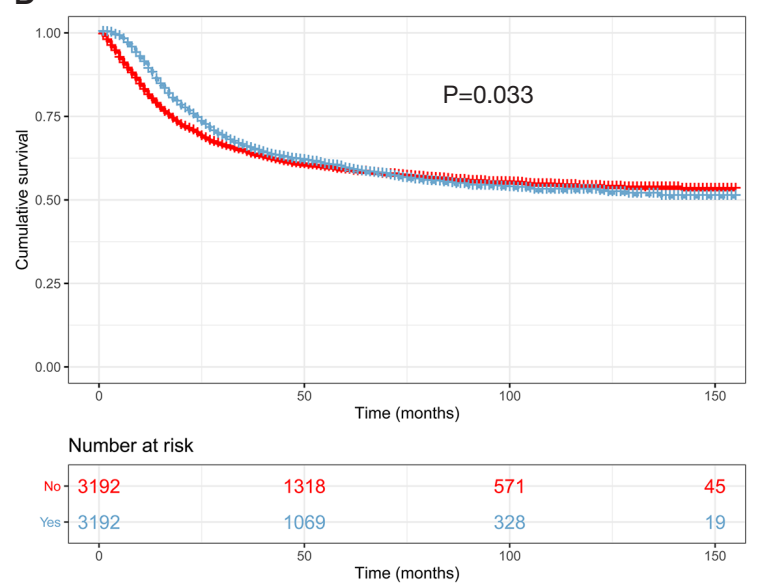

D Adjuvant chemotherapy + No =t+ Yes
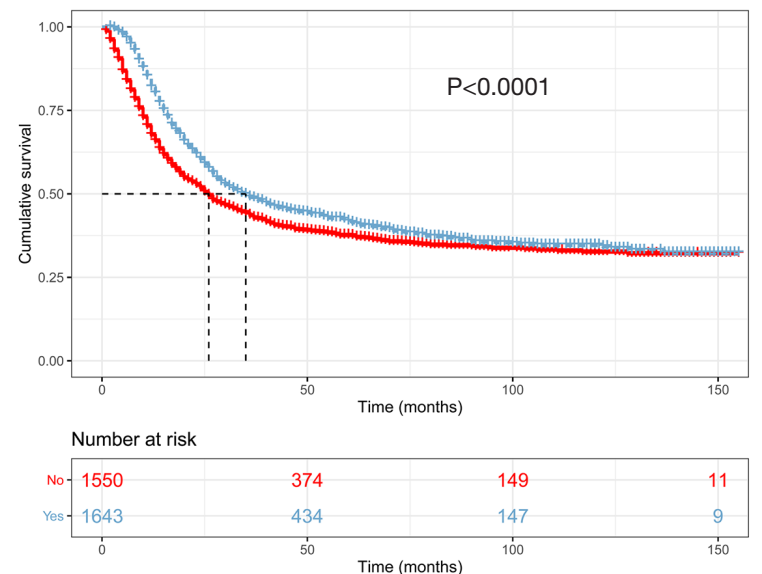

$\mathrm{F}$
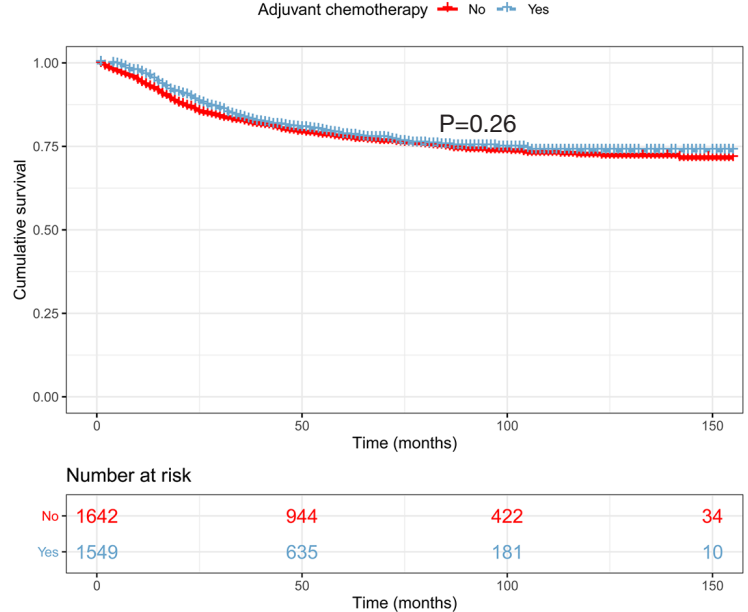

Figure 5 Kaplan-Meier curves of OS and CSS for different risk stratification patients between adjuvant chemotherapy and non-adjuvant chemotherapy. Comparisons of OS (A) and CSS (B) for the entire patients between adjuvant chemotherapy and non-adjuvant chemotherapy. Comparisons of OS (E) and CSS (F) for the low-risk patients between adjuvant chemotherapy and non-adjuvant chemotherapy. Comparisons of OS (C) and CSS (D) for the high-risk patients between adjuvant chemotherapy and non-adjuvant chemotherapy. OS, overall survival; CSS, cancer-specific survival. 
younger MIBC patients. Previously published studies have also noted that elderly patients have greater postoperative comorbidities and mortality, and a worse prognosis than younger MIBC patients (50-53). Although, males have almost a 3 times higher incidence than females $(1,4)$, the prognosis of females patients is generally poorer than that of males $(54,55)$. Gender differences in anatomy, heredity, hormone receptors, tumour biology, diagnosis, and therapy are considered hypotheses about the presumable prognostic impact $(52,56)$. The influence of race on the incidence of BC has not yet been determined. It has been reported that the risk of $\mathrm{BC}$ among Black Americans is much lower compared with White Americans, but the overall survival rate was worse $(57,58)$. Socioeconomic status and access to medicaid insurance can contribute to these prognostic racial disparities $(59,60)$. Several prior studies have found that lymph node counts are independently related to the survival outcomes of BC patients with negative or positive lymph nodes $(22,38,61)$. Some researchers use the LNR as a quality indicator for predicting the prognosis of patients with urothelial $\mathrm{BC}$ and consider it to be superior to the $\mathrm{N}$ staging system $(37,62,63)$. It has been reported that an LNR $>20 \%$ is a poor prognostic factor in patients who underwent $\mathrm{RC}$ after with or without adjuvant chemotherapy $(22,23)$. Tumor size has been validated as an independent prognostic factor in BC patients in previous studies, with large tumors having poor prognostic outcomes $(30,64)$, this was also found in the present study. Additionally, higher $\mathrm{pT}$ and $\mathrm{pN}$ stages are associated with worse prognostic outcomes, which have also been confirmed in previously published studies (65-67). In the present study, demographic and clinicalpathological variables combined with the AJCC TNM staging system, were included in the prognostic nomogram and the risk stratification systems, which may guide more intuitive and accurate clinical decisions-making.

Next, we calculated each patient's total risk score for OS and CSS according to the established nomogram. The lowand high-risk stratification systems for OS and CSS were constructed based on the established nomogram's total risk scores. We evaluated the OS and CSS outcomes of MIBC patients in different prognostic risk stratification systems with and without adjuvant chemotherapy. The outcomes showed that the prognostic nomogram could discriminate between different risk stratification systems for OS and CSS of MIBC patients. However, in the low-risk group, patients receiving adjuvant chemotherapy showed a comparable OS and CSS to those who did not receive adjuvant chemotherapy, indicating that adjuvant chemotherapy may not improve OS and CSS outcomes for low-risk patients. The results are consistent with those of previously published studies $(17,24,27,68)$, where high-risk patients were found to benefit more from adjuvant chemotherapy than lowrisk patients, while patients in the low-risk group should be considered monitored. However, these studies had a common aspect: they categorized each prognostic factor after screening into the same risk level, which may not be suitable for clinical settings. Therefore, we constructed and implemented a simplified and implemented nomogram for assigning points to each prognostic risk factor reasonably. Moreover, DCA is widely applied to evaluate the effectiveness and advantages of predictive models (69-71), and demonstrated high clinical positive net benefits for assessing OS and CSS at different threshold probabilities in the prognostic nomogram.

Admittedly, there are still some potential limitations in our current study. First, despite a large cohort in our study, we excluded a significant number of patients with incomplete demographic characteristics and clinicalpathological information according to the exclusion criteria, which may result in selection bias. Second, although we tried to include all prognostic variables that are significantly associated with MIBC patients into the analyses, not all prognostic risk variables were included in the predictive models. Some positive prognostic variables were not included in our models, and there were no corresponding records obtained in the SEER program, such as smoking, neutrophil-lymphocyte ratio, the specific adjuvant chemotherapy information, surgical marginal status, vascular invasion, and comorbidities, which are significant prognostic factors related to OS and CSS outcomes. Third, many pathological variables included in the prognostic nomogram, which can only be acquired following surgery. Therefore, our hypothesis only applies to the pathological TNM stage, but it is invalid for the clinical TNM stage or post-neoadjuvant TNM stage. Fourth, although the prognostic nomogram and risk stratification systems were established using a large population, the larger independent external cohorts or further randomized clinical and multicenter trials need to be further validated to support our findings. Besides, the absence of data regarding administration of neoadjuvant chemotherapy for cT2a-4N0M0 BC patients in current study. We hope that future studies could establish predictive model and different risk stratification systems for BC patients with neoadjuvant chemotherapy. Finally, the predictions calculated by the nomogram are only for the clinician's decision-making, as 
do not have an absolutely accurate prognostic value.

\section{Conclusions}

In conclusion, we have constructed a predictive model and different risk stratification systems, determine the risk cutoff point, select a population that could benefit from postoperative adjuvant chemotherapy, provide an individualized and accurate reference for the reasonable application of adjuvant chemotherapy. When the patients after RC judged by nomogram are in the low-risk group, adjuvant chemotherapy-induced toxicity and discomfort may be monitored. Our risk score stratification systems may be served as one of a complementary reference for clinical decision making or prospective randomized clinical trial in the future.

\section{Acknowledgments}

We thank AME Editing Service for editing this manuscript. The authors acknowledge the SEER database tumor registries for providing high quality publicly accessible data to researchers.

Funding: This work was supported by the Key Research and Development Program of Zhejiang Province (grant no. 2020C03026).

\section{Footnote}

Reporting Checklist: The authors have completed the TRIPOD reporting checklist. Available at http://dx.doi. org/10.21037/tau-20-960

Peer Review File: Available at http://dx.doi.org/10.21037/ tau-20-960

Conflicts of Interest: All authors have completed the ICMJE uniform disclosure form (available at http://dx.doi. org/10.21037/tau-20-960). All the authors report grants from the Key Research and Development Program of Zhejiang Province, during the conduct of the study. The authors have no other conflicts of interest to declare.

Ethical Statement: The authors are accountable for all aspects of the work in ensuring that questions related to the accuracy or integrity of any part of the work are appropriately investigated and resolved. The study has conformed to the provisions of the Declaration of Helsinki (as revised in 2013).The data analyzed in our current study accessed from SEER database (https://www.seer.cancer. gov/), which is publicly available and all patient data are deidentified. We obtained the permission to access the SEER program with the ID number 17051-Nov2018 via Internet access method. Ethical approval was waived by the local ethics committee.

Open Access Statement: This is an Open Access article distributed in accordance with the Creative Commons Attribution-NonCommercial-NoDerivs 4.0 International License (CC BY-NC-ND 4.0), which permits the noncommercial replication and distribution of the article with the strict proviso that no changes or edits are made and the original work is properly cited (including links to both the formal publication through the relevant DOI and the license). See: https://creativecommons.org/licenses/by-nc-nd/4.0/.

\section{References}

1. Antoni S, Ferlay J, Soerjomataram I, et al. Bladder Cancer Incidence and Mortality: A Global Overview and Recent Trends. Eur Urol 2017;71:96-108.

2. Gray PJ, Fedewa SA, Shipley WU, et al. Use of potentially curative therapies for muscle-invasive bladder cancer in the United States: results from the National Cancer Data Base. Eur Urol 2013;63:823-9.

3. Ferlay J, Steliarova-Foucher E, Lortet-Tieulent J, et al. Cancer incidence and mortality patterns in Europe: estimates for 40 countries in 2012. Eur J Cancer 2013;49:1374-403.

4. Siegel RL, Miller KD, Jemal A. Cancer statistics, 2018. CA Cancer J Clin 2018;68:7-30.

5. Alfred Witjes J, Lebret T, Comperat EM, et al. Updated 2016 EAU Guidelines on Muscle-invasive and Metastatic Bladder Cancer. Eur Urol 2017;71:462-75.

6. Efstathiou JA, Spiegel DY, Shipley WU, et al. Long-term outcomes of selective bladder preservation by combinedmodality therapy for invasive bladder cancer: the MGH experience. Eur Urol 2012;61:705-11.

7. Arcangeli G, Strigari L, Arcangeli S. Radical cystectomy versus organ-sparing trimodality treatment in muscleinvasive bladder cancer: A systematic review of clinical trials. Crit Rev Oncol Hematol 2015;95:387-96.

8. Goossens-Laan CA, Leliveld AM, Verhoeven RH, et al. Effects of age and comorbidity on treatment and survival of patients with muscle-invasive bladder cancer. Int J Cancer 2014;135:905-12. 
9. Hernández-Fernández C, Herranz-Amo F, MoralejoGarate $\mathrm{M}$, et al. Infiltrating bladder cancer: prognostic factors, follow-up and treatment of relapses. Actas Urol Esp 2017;41:352-8.

10. Kim HS, Jeong CW, Kwak C, et al. Adjuvant chemotherapy for muscle-invasive bladder cancer: a systematic review and network meta-analysis of randomized clinical trials. Oncotarget 2017;8:81204-14.

11. Witjes JA, Bruins HM, Cathomas R, et al. European Association of Urology Guidelines on Muscle-invasive and Metastatic Bladder Cancer: Summary of the 2020 Guidelines. Eur Urol 2021;79:82-104.

12. Keegan KA, Zaid HB, Patel SG, et al. Increasing utilization of neoadjuvant chemotherapy for muscleinvasive bladder cancer in the United States. Curr Urol Rep 2014;15:394.

13. Leow JJ, Martin-Doyle W, Rajagopal PS, et al. Adjuvant chemotherapy for invasive bladder cancer: a 2013 updated systematic review and meta-analysis of randomized trials. Eur Urol 2014;66:42-54.

14. Cowan NG, Chen Y, Downs TM, et al. Neoadjuvant chemotherapy use in bladder cancer: a survey of current practice and opinions. Adv Urol 2014;2014:746298.

15. Kim SH, Seo HK, Shin HC, et al. Trends in the Use of Chemotherapy before and after Radical Cystectomy in Patients with Muscle-invasive Bladder Cancer in Korea. J Korean Med Sci 2015;30:1150-6.

16. Booth CM, Siemens DR, Li G, et al. Perioperative chemotherapy for muscle-invasive bladder cancer: A population-based outcomes study. Cancer 2014;120:1630-8.

17. Galsky MD, Stensland KD, Moshier E, et al. Effectiveness of Adjuvant Chemotherapy for Locally Advanced Bladder Cancer. J Clin Oncol 2016;34:825-32.

18. Kwon T, Jeong IG, Lee J, et al. Adjuvant chemotherapy after radical cystectomy for bladder cancer: a comparative study using inverse-probability-of-treatment weighting. J Cancer Res Clin Oncol 2015;141:169-76.

19. Advanced Bladder Cancer (ABC) Meta-analysis Collaboration. Adjuvant chemotherapy in invasive bladder cancer: a systematic review and meta-analysis of individual patient data Advanced Bladder Cancer (ABC) Meta-analysis Collaboration. Eur Urol 2005;48:189-99; discussion 99-201.

20. Cognetti F, Ruggeri EM, Felici A, et al. Adjuvant chemotherapy with cisplatin and gemcitabine versus chemotherapy at relapse in patients with muscle-invasive bladder cancer submitted to radical cystectomy: an Italian, multicenter, randomized phase III trial. Ann Oncol
2012;23:695-700.

21. Sternberg CN, Skoneczna I, Kerst JM, et al. Immediate versus deferred chemotherapy after radical cystectomy in patients with pT3-pT4 or N+M0 urothelial carcinoma of the bladder (EORTC 30994): an intergroup, open-label, randomised phase 3 trial. Lancet Oncol 2015;16:76-86.

22. May M, Herrmann E, Bolenz C, et al. Lymph node density affects cancer-specific survival in patients with lymph node-positive urothelial bladder cancer following radical cystectomy. Eur Urol 2011;59:712-8.

23. Kassouf W, Agarwal PK, Herr HW, et al. Lymph node density is superior to TNM nodal status in predicting disease-specific survival after radical cystectomy for bladder cancer: analysis of pooled data from MDACC and MSKCC. J Clin Oncol 2008;26:121-6.

24. Pouessel D, Bastuji-Garin S, Houédé N, et al. Adjuvant Chemotherapy After Radical Cystectomy for Urothelial Bladder Cancer: Outcome and Prognostic Factors for Survival in a French Multicenter, Contemporary Cohort. Clin Genitourin Cancer 2017;15:e45-e52.

25. Yafi FA, Aprikian AG, Chin JL, et al. Contemporary outcomes of 2287 patients with bladder cancer who were treated with radical cystectomy: a Canadian multicentre experience. BJU Int 2011;108:539-45.

26. Macleod LC, Yabes JG, Yu M, et al. Trends and appropriateness of perioperative chemotherapy for muscleinvasive bladder cancer. Urol Oncol 2019;37:462-9.

27. Froehner M, Koch R, Heberling U, et al. Decreased Overall and Bladder Cancer-Specific Mortality with Adjuvant Chemotherapy After Radical Cystectomy: Multivariable Competing Risk Analysis. Eur Urol 2016;69:984-7.

28. Cronin KA, Ries LA, Edwards BK. The Surveillance, Epidemiology, and End Results (SEER) Program of the National Cancer Institute. Cancer 2014;120 Suppl 23:3755-7.

29. Dong F, Shen Y, Gao F, et al. Nomograms to Predict Individual Prognosis of Patients with Primary Small Cell Carcinoma of the Bladder. J Cancer 2018;9:1152-64.

30. Huang C, Zhou W, Song P, et al. Comparison of different prognostic models for predicting cancer-specific survival in bladder transitional cell carcinoma. Future Oncol 2019;15:851-64.

31. Maruf M, Sidana A, Purnell S, et al. Lymph node dissection during radical cystectomy following prior radiation therapy: results from the SEER database. Int Urol Nephrol 2018;50:257-62.

32. Abdollah F, Sun M, Schmitges J, et al. Stage-specific 
impact of pelvic lymph node dissection on survival in patients with non-metastatic bladder cancer treated with radical cystectomy. BJU Int 2012;109:1147-54.

33. Konety BR, Joslyn SA, O'Donnell MA. Extent of pelvic lymphadenectomy and its impact on outcome in patients diagnosed with bladder cancer: analysis of data from the Surveillance, Epidemiology and End Results Program data base. J Urol 2003;169:946-50.

34. Herr H, Lee C, Chang S, et al. Standardization of radical cystectomy and pelvic lymph node dissection for bladder cancer: a collaborative group report. J Urol 2004;171:1823-8; discussion 1827-8.

35. Wright JL, Lin DW, Porter MP. The association between extent of lymphadenectomy and survival among patients with lymph node metastases undergoing radical cystectomy. Cancer 2008;112:2401-8.

36. Stein JP, Cai J, Groshen S, et al. Risk factors for patients with pelvic lymph node metastases following radical cystectomy with en bloc pelvic lymphadenectomy: concept of lymph node density. J Urol 2003;170:35-41.

37. Herr HW. Superiority of ratio based lymph node staging for bladder cancer. J Urol 2003;169:943-5.

38. Morgan TM, Barocas DA, Penson DF, et al. Lymph node yield at radical cystectomy predicts mortality in node-negative and not node-positive patients. Urology 2012;80:632-40.

39. Austin PC. An Introduction to Propensity Score Methods for Reducing the Effects of Confounding in Observational Studies. Multivariate Behav Res 2011;46:399-424.

40. Kamarudin AN, Cox T, Kolamunnage-Dona R. Timedependent ROC curve analysis in medical research: current methods and applications. BMC Med Res Methodol 2017;17:53.

41. Vickers AJ, Elkin EB. Decision curve analysis: a novel method for evaluating prediction models. Med Decis Making 2006;26:565-74.

42. Iasonos A, Schrag D, Raj GV, et al. How to build and interpret a nomogram for cancer prognosis. J Clin Oncol 2008;26:1364-70.

43. Stenzl A, Cowan NC, De Santis M, et al. Treatment of muscle-invasive and metastatic bladder cancer: update of the EAU guidelines. Eur Urol 2011;59:1009-18.

44. Bensalah K, Roupret M, Xylinas E, et al. The survival benefit of lymph node dissection at the time of removal of kidney, prostate and urothelial carcinomas: what is the evidence? World J Urol 2013;31:1369-76.

45. Tilki D, Brausi M, Colombo R, et al. Lymphadenectomy for bladder cancer at the time of radical cystectomy. Eur
Urol 2013;64:266-76.

46. Gandaglia G, Varda B, Sood A, et al. Short-term perioperative outcomes of patients treated with radical cystectomy for bladder cancer included in the National Surgical Quality Improvement Program (NSQIP) database. Can Urol Assoc J 2014;8:E681-7.

47. Shabsigh A, Korets R, Vora KC, et al. Defining early morbidity of radical cystectomy for patients with bladder cancer using a standardized reporting methodology. Eur Urol 2009;5 5:164-74.

48. Grossman HB, Natale RB, Tangen CM, et al. Neoadjuvant chemotherapy plus cystectomy compared with cystectomy alone for locally advanced bladder cancer. N Engl J Med 2003;349:859-66.

49. Droller MJ. Neoadjuvant cisplatin, methotrexate, and vinblastine chemotherapy for muscle-invasive bladder cancer: a randomised controlled trial. J Urol 2000;163:1602-3.

50. Rink M, Dahlem R, Kluth L, et al. Older patients suffer from adverse histopathological features after radical cystectomy. Int J Urol 2011;18:576-84.

51. Fontana PP, Gregorio SA, Rivas JG, et al. Perioperative and survival outcomes of laparoscopic radical cystectomy for bladder cancer in patients over 70 years. Cent European J Urol 2015;68:24-9.

52. Shariat SF, Sfakianos JP, Droller MJ, et al. The effect of age and gender on bladder cancer: a critical review of the literature. BJU Int 2010;105:300-8.

53. Fairey AS, Kassouf $W$, Aprikian AG, et al. Age $>/=80$ years is independently associated with survival outcomes after radical cystectomy: results from the Canadian Bladder Cancer Network Database. Urol Oncol 2012;30:825-32.

54. Tracey E, Roder D, Luke C, et al. Bladder cancer survivals in New South Wales, Australia: why do women have poorer survival than men? BJU Int 2009;104:498-504.

55. Mungan NA, Aben KK, Schoenberg MP, et al. Gender differences in stage-adjusted bladder cancer survival. Urology 2000;5 5:876-80.

56. Fajkovic H, Halpern JA, Cha EK, et al. Impact of gender on bladder cancer incidence, staging, and prognosis. World J Urol 2011;29:457-63.

57. Prout GR, Jr., Wesley MN, McCarron PG, et al. Survival experience of black patients and white patients with bladder carcinoma. Cancer 2004;100:621-30.

58. Scosyrev E, Noyes K, Feng C, et al. Sex and racial differences in bladder cancer presentation and mortality in the US. Cancer 2009;115:68-74.

59. Halpern MT, Ward EM, Pavluck AL, et al. Association 
of insurance status and ethnicity with cancer stage at diagnosis for 12 cancer sites: a retrospective analysis. Lancet Oncol 2008;9:222-31.

60. Sung JM, Martin JW, Jefferson FA, et al. Racial and Socioeconomic Disparities in Bladder Cancer Survival: Analysis of the California Cancer Registry. Clin Genitourin Cancer 2019;17:e995-1002.

61. May M, Herrmann E, Bolenz C, et al. Association between the number of dissected lymph nodes during pelvic lymphadenectomy and cancer-specific survival in patients with lymph node-negative urothelial carcinoma of the bladder undergoing radical cystectomy. Ann Surg Oncol 2011;18:2018-25.

62. Osawa T, Abe T, Shinohara N, et al. Role of lymph node density in predicting survival of patients with lymph node metastases after radical cystectomy: a multi-institutional study. Int J Urol 2009;16:274-8; discussion 278.

63. Wiesner C, Salzer A, Thomas C, et al. Cancer-specific survival after radical cystectomy and standardized extended lymphadenectomy for node-positive bladder cancer: prediction by lymph node positivity and density. BJU Int 2009;104:331-5.

64. Youssef RF, Lotan Y. Predictors of outcome of nonmuscle-invasive and muscle-invasive bladder cancer. ScientificWorldJournal 2011;11:369-81.

Cite this article as: Tian J, Sun J, Fu G, Xu Z, Chen X, Shi $\mathrm{Y}$, Jin B. Population-based outcome of muscle-invasive bladder cancer following radical cystectomy: who can benefit from adjuvant chemotherapy? Transl Androl Urol 2021;10(1):356-373. doi: $10.21037 /$ tau-20-960
65. Bochner BH, Kattan MW, Vora KC. Postoperative nomogram predicting risk of recurrence after radical cystectomy for bladder cancer. J Clin Oncol 2006;24:3967-72.

66. Margulis V, Lotan Y, Montorsi F, et al. Predicting survival after radical cystectomy for bladder cancer. BJU Int 2008;102:15-22.

67. Zehnder P, Studer UE, Daneshmand S, et al. Outcomes of radical cystectomy with extended lymphadenectomy alone in patients with lymph node-positive bladder cancer who are unfit for or who decline adjuvant chemotherapy. BJU Int 2014;113:554-60.

68. Shimizu F, Muto S, Taguri M, et al. Effectiveness of platinum-based adjuvant chemotherapy for muscle-invasive bladder cancer: A weighted propensity score analysis. Int J Urol 2017;24:367-72.

69. Van Calster B, Wynants L, Verbeek JFM, et al. Reporting and Interpreting Decision Curve Analysis: A Guide for Investigators. Eur Urol 2018;74:796-804.

70. Hernandez JM, Tsalatsanis A, Humphries LA, et al. Defining optimum treatment of patients with pancreatic adenocarcinoma using regret-based decision curve analysis. Ann Surg 2014;259:1208-14.

71. Fitzgerald M, Saville BR, Lewis RJ. Decision curve analysis. JAMA 2015;313:409-10. 
Raw Treated

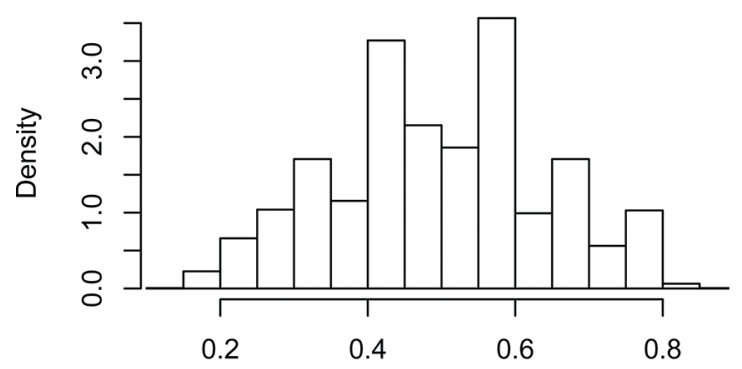

Propensity Score

Raw Control

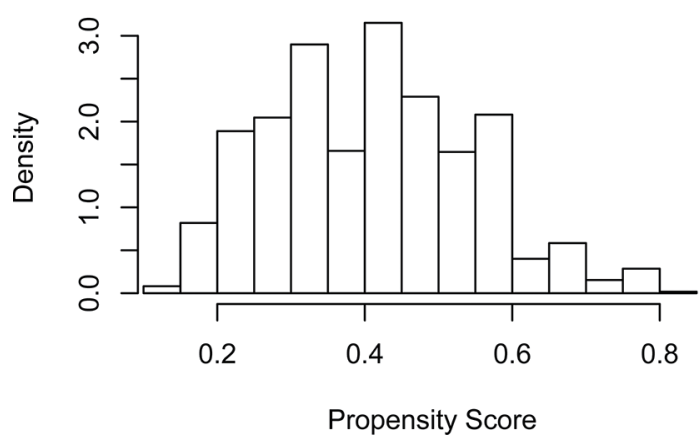

Matched Treated

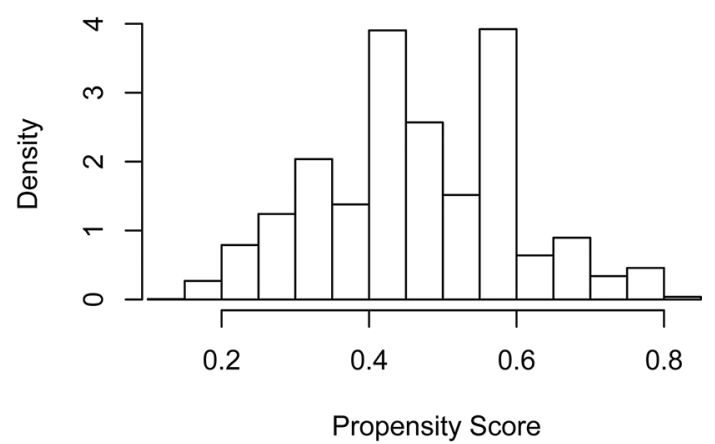

Matched Control

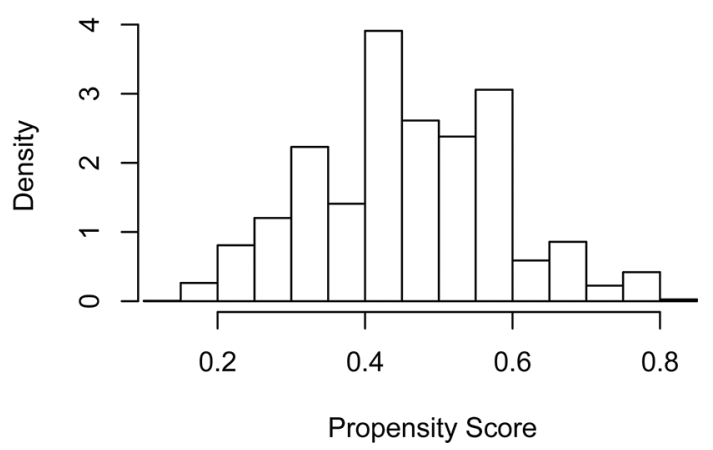

Figure S1 Histograms of the propensity scores before and after matching.
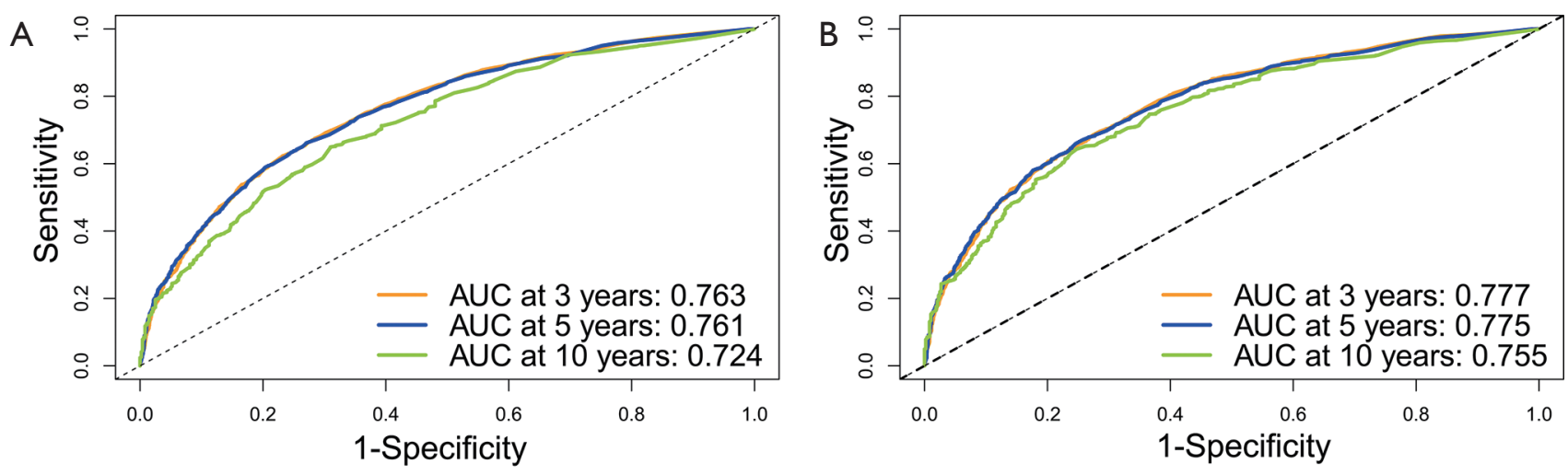

C
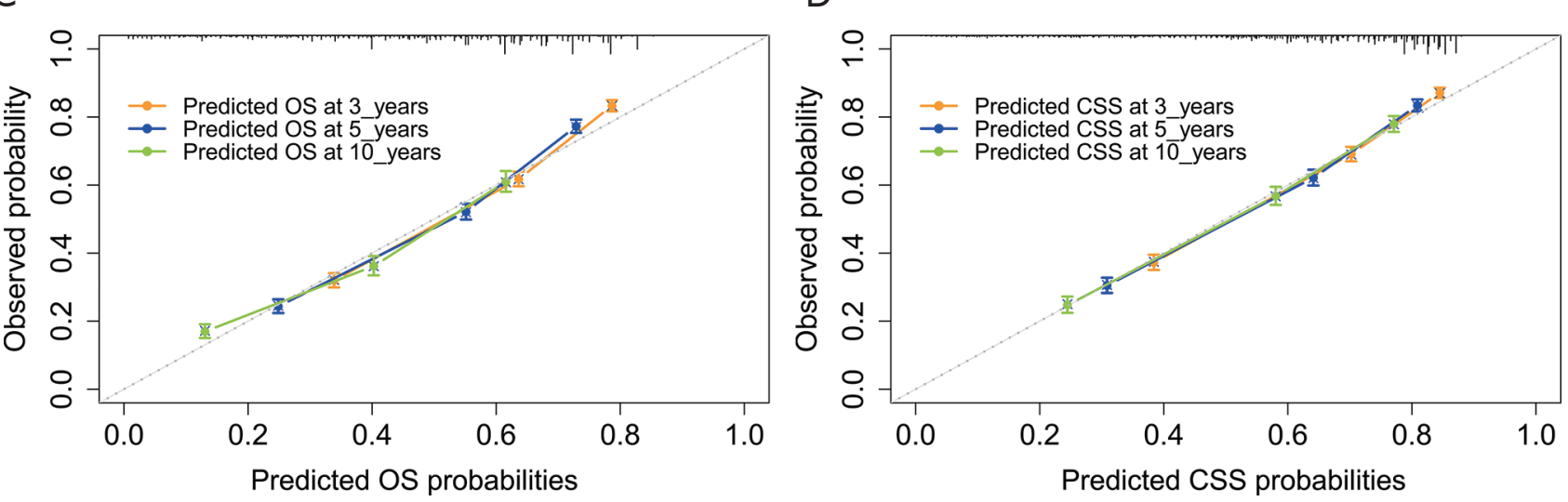

Figure S2 The discrimination and calibration curves of nomogram for 3-, 5- and 10-year OS and CSS. (A) and (B) show the ROC curves of the 3-, 5-, and 10-year OS and CSS nomogram, respectively. C and D show the calibration curves of 3-, 5-, and 10-year OS and CSS nomogram, respectively. The 45-degree dashed line represents a perfect match between the nomogram-predicted survival (X-axis) and actual survival (Y-axis). The perpendicular line represents $95 \%$ confidence intervals (CI) of actual survival. AUC, area under the curve; OS, overall survival; CSS, cancer-specific survival; ROC, receiver operating characteristic. 